\title{
PHOTOABSORPTION AND PHOTOPRODUCTION ON NUCLEI IN THE RESONANCE REGION
}

\author{
S. SCHADMAND \\ Institut für Kernhysik \\ Forschungszentrum Jülich \\ D-52425 Jülich, Germany \\ E-mail: s.schadmand@fz-juelich.de
}

\begin{abstract}
Inclusive studies of nuclear photoabsorption have provided clear evidence of medium modifications in the properties of hadrons. However, the results have not been explained in a model independent way. A deeper understanding of the situation is expected from a detailed comparison of meson photoproduction from nucleons and from nuclei in exclusive reactions. Recent experimental results are presented.
\end{abstract}

\section{Introduction}

Current issues in the understanding of the strong interaction address the structure of hadrons, consisting of quarks and gluons, as the building blocks of matter. Central challenges concern the questions why quarks are confined within hadrons and how hadrons are constructed from their constituents. One goal is to find the connection between the parton degrees of freedom and the low energy structure of hadrons leading to the study of the hadron excitation spectrum and the search for exotic states, like glueballs or hybrid states. An approach related to the question of the origin of hadron masses is the search for modifications of hadron properties in the nuclear medium. The underlying question is the origin of hadron masses in the context of chiral symmetry breaking. Evidence for such effects has been searched for in many experiments. In this contribution, photoabsorption and meson photoproduction on nuclei are discussed.

\section{Nuclear Photoabsorption}

Photoabsorption experiments on the free nucleon demonstrate the complex structure of the nucleon and its excitation spectrum, as shown in Fig. 1. The lowest-lying peak is the $\Delta(1232)$ resonance and is prominently excited 
by incident photons of $0.2-0.5 \mathrm{GeV}$. The following group of resonances, $\mathrm{P}_{11}(1440), \mathrm{D}_{13}(1520)$, and $\mathrm{S}_{11}(1535)$, is called the second resonance region $\left(\mathrm{E}_{\gamma}=0.5-0.9 \mathrm{GeV}\right)$, a third resonance region is visible. The observed resonance structures have been studied using their decay via light mesons, showing that the photoabsorption spectrum can be explained by the sum of $\pi, \pi \pi$ and $\eta$ production cross sections.

Fig. 1 also shows the nuclear photoabsorption cross section per nucleon as an average over the nuclear systematics ${ }^{1}$. The $\Delta$ resonance is broadened

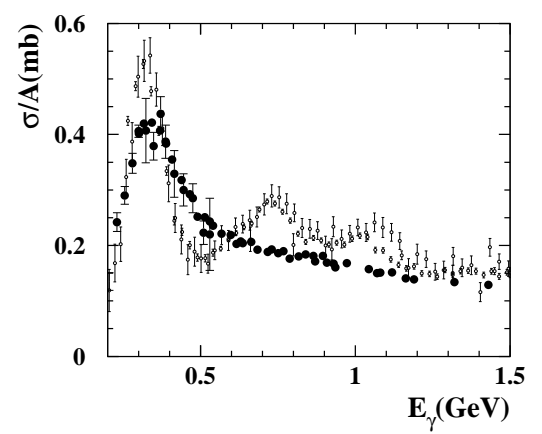

Figure 1. Nuclear photoabsorption cross section per nucleon as an average over the nuclear systematics ${ }^{1}$ (full symbols) compared to the absorption on the proton ${ }^{2}$ (open symbols).

and slightly shifted while the second and higher resonance regions seem to have disappeared.

Mosel et al. ${ }^{3,4,5}$ have argued that an in-medium broadening of the $\mathrm{D}_{13}(1520)$ resonance is a likely cause of the suppressed photoabsorption cross section. Hirata et al. ${ }^{6}$ see a change of the interference effects in the nuclear medium as one of the most important reasons for the suppression of the resonance structure. However, the absence of resonance structure in nuclear photoabsorption has not been explained in a model-independent way. A deeper understanding of the situation is anticipated from the experimental study of meson photoproduction on nucleons embedded in nuclei in comparison to studies on the free nucleon.

\section{Meson Production in the Second Resonance Region}

In the second resonance region, double pion production aims at the resonances $\mathrm{D}_{13}(1520)$ and $\mathrm{P}_{11}(1440)$ while $\eta$ production is characteristic for the $\mathrm{S}_{11}(1535)$ resonance. The three resonances in the second resonance region 
decay to roughly $50 \%$ via single pion emission. The most trivial medium modification is the broadening of the excitation functions due to Fermi motion. The decay of the resonances is further modified by Pauli-blocking of final states, which reduces the resonance widths. In addition, decay channels like $\mathrm{N}^{\star} \mathrm{N} \rightarrow \mathrm{NN}$ cause collisional broadening. Both effects could cancel to some extent.
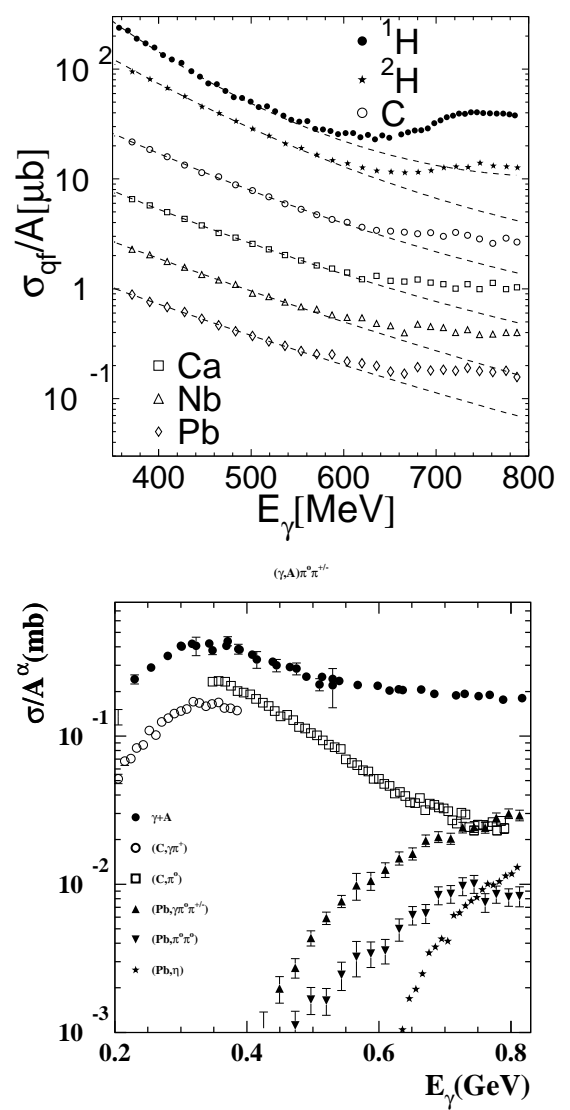

Figure 2. Left: Total cross section per nucleon for single $\pi^{\circ}$ photoproduction in the second resonance region for the nucleon and for nuclei. The scale corresponds to the proton data, the other data are scaled down by factors 2,4,8,16,32, respectively. The dashed curves are fits to the data in the energy range 350-550 MeV. Right: Status of the decomposition of nuclear photoabsorption into meson production channels (scaled with $\left.\mathrm{A}^{\alpha}, \alpha=2 / 3\right)$. Small open circles are the average nuclear photoabsorption cross section per nucleon $(\alpha=1)^{1}$. Meson production data are from $7,8,9,10,11,12$. 
An attempt to study the in-medium properties of the $\mathrm{D}_{13}$ resonance was undertaken with a measurement of quasifree single $\pi^{\circ}$ photoproduction ${ }^{8}$ which, on the free nucleon, is almost exclusively sensitive to the $\mathrm{D}_{13}$ resonance. The left panel of Fig. 2 summarizes the results. Strong quenching of the $\mathrm{D}_{13}$-resonance structure is found for the deuteron with respect to the nucleon. However, an indication of a broadening or a suppression of the $\mathrm{D}_{13}$ structure in heavy nuclei is not observed. Model predictions agree with the pion photoproduction data only under the assumption of a strong broadening of the resonance, other effects seem to be missing in the models. This casts doubt on the interpretation of the total photoabsorption data via resonance broadening. In contrast to the case of total photoabsorption, the second resonance bump remains visible. However, exclusive reaction channels are dominated by the nuclear surface region where in-medium effects are smaller. Furthermore, as discussed in ${ }^{13}$, resonance broadening effects are even more diluted for reactions which do not contribute to the broadening, due to the averaging over the nuclear volume.

The right panel of Fig. 2 shows the status of the decomposition of nuclear photoabsorption into meson production channels. The available experimental meson cross sections are exclusive measurements, investigating quasifree production. The purely charged final states have not been measured. However, it can be inferred from the existing data that the sum of the cross sections would not reproduce the flat shape of the total photoabsorption from nuclei. In a recent compilation ${ }^{14}$, it is observed that the current results indicate large differences between quasifree meson production from the nuclear surface and non-quasifree components. The quasifree part does not show a suppression of the resonance structures in the second resonance region. However, resonance structures seem absent in the non-quasifree meson production which has larger contributions from the nuclear volume.

\section{4. $\omega$ Mesons in the nuclear Medium}

The photoproduction of $\omega$ mesons on nuclei has been investigated using the Crystal Barrel/TAPS experiment at the ELSA tagged photon facility in Bonn ${ }^{15}$. The aim is to study possible in-medium modifications of the $\omega$ meson via the reaction $\gamma+A \rightarrow \omega+X \rightarrow \pi^{\circ} \gamma+X^{\prime}$. A number of theoretical models predict a mass shift of the omega meson in the nuclear medium, for references see ${ }^{16,15}$. An excellent recent review discusses nucleon and hadron structure changes in the nuclear medium within the quark-meson coupling (QMC) model predicting a reduction in the omega mass in nuclei 
17. Experimentally, results obtained for $\mathrm{Nb}$ are compared to a reference measurement on a $\mathrm{LH}_{2}$ target. The left panel of Fig. 3 shows the $\pi^{\circ} \gamma$
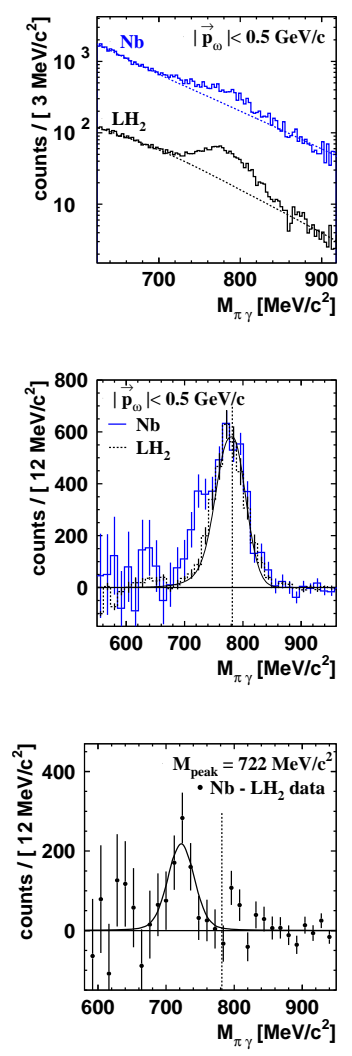

Figure 3. Left panel: Inclusive $\pi^{\circ} \gamma$ invariant mass spectra for $\omega$ momenta less than 500 $\mathrm{MeV} / \mathrm{c}$. Upper histogram: $\mathrm{Nb}$ data, lower histogram: $\mathrm{LH}_{2}$ target reference measurement. The dashed lines indicate fits to the respective background. Middle panel: $\pi^{\circ} \gamma$ invariant mass for the $\mathrm{Nb}$ data (solid histogram) and $\mathrm{LH}_{2}$ data (dashed histogram) after background subtraction. The error bars show statistical uncertainties only. The solid curve represents the simulated line shape for the $\mathrm{LH}_{2}$ target. Right panel: In-medium decays of $\omega$ mesons along with a Voigt fit to the data. The vertical line indicates the vacuum $\omega$ mass of $782 \mathrm{MeV} / \mathrm{c}^{2}$.

invariant mass distribution without further cuts except for a three momentum cutoff of $\left|\vec{p}_{\omega}\right|<500 \mathrm{MeV} / \mathrm{c}$. The dominant background source is two pion production where one of the four photons escapes the detection. This 
probability was determined by Monte Carlo simulations to be $14 \%$. The resulting three photon final state is not distinguishable from the $\omega \rightarrow \pi^{\circ} \gamma$ invariant mass. These decays are eliminated by matching the right hand part of the $\mathrm{Nb}$ invariant mass spectrum to the $\mathrm{LH}_{2}$ data (see central panel of Fig. 3) and by subtracting the two spectra from each other. For this normalization the integral of the undistorted spectrum corresponds to $75 \%$ of the counts in the Nb spectrum. This is in good agreement with a theoretical prediction obtained from a transport code calculation ${ }^{18,19}$. There, about $16 \%$ of the total decays are predicted to occur inside the nuclear medium $\left(\rho>0.1 \cdot \rho_{0}\right)$ without final state interaction (FSI) and $3 \%$ of the events are distorted due to FSI in the mass range of $0.6 \mathrm{GeV} / \mathrm{c}^{2}<\mathrm{M}_{\pi^{\circ} \gamma}<0.9 \mathrm{GeV} / \mathrm{c}^{2}$. In addition, $9 \%$ of the events are moved towards lower masses due to the $\Delta$ decay kinematics. The right panel of Fig. 3 shows the invariant mass distribution obtained after background subtraction. The expected superposition of decays outside of the nucleus is observed at the nominal vacuum mass with decays occurring inside the nucleus, responsible for the shoulder towards lower invariant masses. The high mass part of the $\omega$ mass signal appears to be identical for the $\mathrm{Nb}$ and $\mathrm{LH}_{2}$ targets, indicating that this part is dominated by $\omega$ meson decays in vacuum.

A difference in the line shape for the two data samples is not observed for recoiling, long-lived mesons $\left(\pi^{\circ}, \eta\right.$ and $\left.\eta^{\prime}\right)$, which decay outside of the nucleus. However, for $\omega$ mesons produced on the $\mathrm{Nb}$ target a significant enhancement towards lower masses is found. For momenta less than $500 \mathrm{MeV} / \mathrm{c}$ an in-medium $\omega$ meson mass of $\mathrm{M}_{\text {medium }}=\left[722_{-2}^{+2} \text { (stat) }\right)_{-5}^{+35}$ (syst) $] \mathrm{MeV} / \mathrm{c}^{2}$ has been deduced at an estimated average nuclear density of $0.6 \rho_{0}$.

\section{Summary}

The systematic study of total cross sections for single $\pi^{\circ}, \eta$, and $\pi \pi$ production over a series of nuclei has not provided an obvious hint for a depletion of resonance yield. The observed reduction and change of shape in the second resonance region are mostly as expected from absorption effects, Fermi smearing and Pauli blocking, and collisional broadening. The sum of experimental meson cross sections for neutral and mixed charged states between 400 and $800 \mathrm{MeV}$ demonstrates the persistence of the second resonance bump when at least one neutral meson is observed. It has to be concluded that the medium modifications leading to the depletion of cross section in nuclear photoabsorption are a subtle interplay of effects. Their 
investigation and the rigorous comparison to theoretical models requires the detailed study of differential cross sections and a deeper understanding of meson production in the nuclear medium. The recent experimental investigation of $\omega$ photoproduction from nuclei has been presented as one detailed study of medium modification of hadrons. First evidence for a lowering of the $\omega$ mass in the nuclear medium has been observed.

\section{References}

1. V. Muccifora, et al., Phys. Rev. C60 (1999) 064616.

2. K. Hagiwara, et al., Phys. Rev. D66 (2002) 010001.

3. U. Mosel, Prog. Part. Nucl. Phys. 42 (1999) 163-176.

4. J. Lehr, M. Effenberger, U. Mosel, Nucl. Phys. A671 (2000) 503-531.

5. M. Effenberger, A. Hombach, S. Teis, U. Mosel, Nucl. Phys. A614 (1997) 501-520.

6. M. Hirata, N. Katagiri, K. Ochi, T. Takaki, Phys. Rev. C66 (2002) 014612.

7. J. Arends, et al., Z. Phys. A305 (1982) 205.

8. B. Krusche, et al., Phys. Rev. Lett. 86 (2001) 4764-4767.

9. M. Roebig-Landau, et al., Phys. Lett. B373 (1996) 45-50.

10. H. Yamazaki, et al., Nucl. Phys. A670 (2000) 202-205.

11. S. Janssen, PhD thesis, University of Giessen (2002).

12. S. Schadmand, Habilitation thesis, University of Giessen (2005).

13. J. Lehr, U. Mosel, Phys. Rev. C64 (2001) 042202.

14. B. Krusche, et al., Eur. Phys. J. A22 (2004) 347-351.

15. D. Trnka, et al., Phys. Rev. Lett. 94 (2005) 192303.

16. J. G. Messchendorp, A. Sibirtsev, W. Cassing, V. Metag, S. Schadmand, Eur. Phys. J. A11 (2001) 95-103.

17. K. Saito, K. Tsushima, A. W. Thomas, hep-ph/0506314.

18. P. Muhlich, T. Falter, U. Mosel, Eur. Phys. J. A20 (2004) 499-508.

19. P. Muhlich, U. Mosel, private ecommunication. 